\title{
Aprendizaje por descubrimiento de la crisis de refugiados y de los inmigrantes en el grado de Marketing de la Universidad Francisco de Vitoria
}

\author{
Miguel Osorio (1), Raquel Ayestarán (2) y M. Cristina Fuentes-Lara (1) * \\ (1) Cátedra de Inmigración de la Universidad Francisco de Vitoria, Carretera Pozuelo-Majadahonda km. 1,8, Pozuelo de \\ Alarcón, Madrid - España. (correo-e:m.osorio@ufv.es; cristina.fuentes@ufv.es) \\ (2) Facultad de Jurídicas y Empresariales. Grado de Marketing de la Universidad Francisco de Vitoria, Carretera \\ Pozuelo-Majadahonda km. 1,8, Pozuelo de Alarcón, Madrid - España. (correo-e: r.ayestaran@ufv.es)
}

*Autor a quien debe ser enviada la correspondencia

Recibido Dic. 23, 2019; Aceptado Feb. 18, 2020; Versión final Abr. 2, 2020, Publicado Ago. 2020

\begin{abstract}
Resumen
El objetivo de esta investigación es indagar sobre el aprendizaje por descubrimiento en el estudiante universitario, para comprobar que esas metodologías educativas también eran eficaces en la enseñanza del marketing y, además, podían responder a un reto social. Para ello, se ha realizó una investigación en la que, el Grado en Marketing y la Cátedra de investigación sobre Inmigración de la Universidad Francisco de Vitoria, se unieron para aplicar las metodologías del aprendizaje con 60 estudiantes que cursaban fundamentos del marketing en los cursos 2017-2018 y 2018-2019. Los estudiantes, organizados por equipos, primero investigaron una realidad social que desconocían y sobre la que tenían prejuicios. El método de investigación ha sido cuantitativo, por medio de un cuestionario y la técnica de análisis fue univariante, bivariante y multivariante. Los resultados de la investigación muestran que el estudiante está más satisfecho y valora más positivamente la pedagogía de aprendizaje por descubrimiento. Las principales conclusiones alumbran que los estudiantes manifestaron su comprensión de la dignidad de las personas refugiadas y migrantes y, su satisfacción por haber descubierto que podían ayudarlas con sus conocimientos de marketing.
\end{abstract}

\section{Learning by discovery of the refugee crisis and immigrants in the Marketing degree of Francisco de Vitoria University}

\begin{abstract}
The objective of this research is to investigate discovery learning in the university student, to verify that these educational methodologies were also effective in teaching marketing and, in addition, could respond to a social challenge. For this, an investigation was carried out in which, the Degree in Marketing and the Research Chair on Immigration of the Francisco de Vitoria University, came together to apply the learning methodologies with 60 students who were studying marketing fundamentals in the 2017 courses -2018 and 2018-2019. The students, organized in teams, first investigated a social reality that they were unaware of and prejudiced about. The research method was quantitative, using a questionnaire, and the analysis technique was univariate, bivariate, and multivariate. The research results show that the student is more satisfied and values the discovery learning pedagogy more positively. The main conclusions show that the students expressed their understanding of the dignity of refugees and migrants and their satisfaction at having discovered that they could help them with their marketing knowledge.
\end{abstract}

Keywords: learning by discovery; cooperative; social marketing; refugees; immigrants. 


\section{INTRODUCCIÓN}

La investigación centrada en el aprendizaje por descubrimiento ha sido muy prolífica en las últimas décadas (Nava y Rueda, 2014; Farieta, 2013; Aparicio-Gervás y Delgado, 2011). Sin embargo, su aplicación docente ha sido más discreta. De hecho, esta problemática ya la advertía Bruner (1961), para quien la teoría de la reminiscencia era un excelente sistema pedagógico, pero que la implantación en la educación formal y reglada era un reto. Las investigaciones de Arias $(2014,2010)$ indicaban que la praxis del aprendizaje por descubrimiento en temáticas sociales generaba estímulos mayores entre los estudiantes. En el caso de este artículo se optó por unir el marketing social (Sajid, 2016; Valle-Robles, 2017) y la inmigración (Ager y Strang, 2008; Davies y Batchelor, 2017; Crawley y Skleparis, 2018).

La experiencia de formación universitaria que exponemos en este artículo, parte de una alianza interdepartamental en la Universidad Francisco de Vitoria (UFV) en Madrid (España). En concreto, los responsables del Grado en Marketing y de la Cátedra de Inmigración tenían como propósito que sus estudiantes adquirieses los conocimientos del marketing de una forma completa y motivadora. El programa acordado por ambos departamentos consistió en dos propuestas a los estudiantes del Grado de Marketing. La primera propuesta se desarrolló con el alumnado de primero en el curso 2017-2018, y que consistía en que los estudiantes se tenían que organizar en equipos para realizar un trabajo de investigación sobre la crisis de los refugiados derivada del conflicto bélico de Siria. La segunda propuesta se realizó con los estudiantes de primer curso del Grado del Marketing en el curso 2018-2019. En este caso, la actividad siguió el mismo proceso que el año anterior, con la salvedad de que el aprendizaje por descubrimiento se llevaría a cabo con una temática diferente, es decir, la situación de los jóvenes latinoamericanos en Madrid.

Los estudiantes debían profundizar sobre una realidad que sólo conocían superficialmente a través de los informativos de los medios de comunicación social (Morales-Maure et al., 2018). Tras esa primera fase de estudio, debían utilizar su aprendizaje en la elaboración de una campaña de marketing cuyo objetivo era proponer una mayor comprensión y solidaridad con las personas que sufrían la crisis de los refugiados y en la situación de los jóvenes latinoamericanos en Madrid. El desarrollo de las campañas fue valorado por un equipo de profesorado y expertos en marketing social; y por investigadores de la Cátedra de inmigración que, determinaron la alta calidad de los trabajos presentados tanto por el contenido como por la aplicación de las técnicas de marketing aprendidas en clase anteriormente. La hipótesis de la investigación es que "el aprendizaje por descubrimiento genera en los estudiantes universitario una mayor implicación y una valoración positiva de la temática que están estudiando y / o aplicando". Es por ello, que el objetivo general de la investigación es indagar sobre el aprendizaje por descubrimiento en los estudiantes universitarios, para comprobar que esas metodologías educativas también eran eficaces en la enseñanza del marketing y, además, podían responder a un reto social. La conceptualización teórica de esta investigación se cimenta en dos arquetipos conceptuales: el marketing social como una estrategia para visibilizar las causas sociales y el aprendizaje por descubrimiento como metodología didáctica.

El marketing social como incentivo para las causas sociales. Si partimos de la definición de marketing propia de la American Marketing Association (1985), el marketing es el proceso de planificación y ejecución del concepto, precio, promoción y distribución de ideas de bienes y servicios para crear intercambios que satisfagan los objetivos del individuo y de la organización. A esta definición se la ha querido modernizar encuadrándolos en un mercado ampliamente competitivo y globalizado. De hecho, autores como Cova y Badot (1995) se alejan de la visión economicista del marketing y profundizan en la idea de poner los productos con sentido en la sociedad. Defienden más un intercambio social que la venta por encima de todo. Tal es así que, para un teórico del marketing como Sajid (2016) es la forma democrática de organizar la sociedad la que posibilita un marketing, donde la elección de los productos por los individuos se haga desde una amplia posición de libertad.

En este punto es donde emerge el marketing social que es una tipología de marketing que consiste en añadir en el proceso de intercambio a un nuevo beneficiario, específicamente a una causa social (Olarte et al., 2011). Sin embargo, añadir un beneficiario no significa que ni el cliente ni la empresa dejen de beneficiarse de esa operación, sino que comparten su satisfacción-interés por el producto y sus contraprestaciones, con el de un grupo social necesitado o una causa social merecedora de apoyo (Blom, 2000). En 1970, y con participación de teóricos y prácticos del marketing general, se empieza a desarrollar el marketing social, tratando de adaptar y transferir los elementos comerciales a las actividades dedicadas a defender los intereses de la sociedad (Valle-Robles, 2017). Kotler y Zaltman (1971) conceptualizan el marketing social como "el diseño implantación y control de programas que buscan incrementar la aceptabilidad de una idea social o prácticas en grupos" (p. 19), rechazando comportamientos potencialmente indeseables para los grupos o las causas objetivo (Kotler y Lee, 2008). De tal forma, que el objetivo del marketing social es lograr el compromiso de los consumidores hacia un interés social, generando beneficios de forma directa a la empresa (Kotler, 2003). En la misma línea Andreasen (1995) señala que el marketing social es la "adaptación del marketing comercial a los programas diseñados para influir en el comportamiento voluntario de la audiencia meta, con el fin, de mejorar su bienestar y el de la 
sociedad en general, por medio del uso de la tecnología del marketing comercial en los programas sociales" (p. 3). El marketing social mantiene las cuatro Ps del marketing social, es decir, Producto, Precio, Plaza y Promoción; añadiéndole tres Ps específicas del sector social como son Proceso, Presentación y Personal (Pérez, 2004). Precisamente, de la interacción de las siete Ps del marketing social es de donde ha partido el análisis de este artículo, aplicado al aprendizaje por descubrimiento.

El aprendizaje por descubrimiento. El aprendizaje por descubrimiento tiene marcados sus comienzos, como formulación de teoría, en la filosofía socrática (Arias, 2014). De tal forma, que la metodología de la filosofía socrática consistía en la formulación de preguntas por parte del profesor, en el este caso Sócrates, para que los discípulos reflexionaran sobre ellas (Arias, 2010). De hecho, uno de sus discípulos, Platón profundizó sobre esta metodología y diseñó la teoría de la reminiscencia (Farieta, 2013). Posteriormente, autores relevantes en investigación educativa como Rousseau (1971), Dewey (1938), Bruner (1956), Piaget (1983), y, Goodnow y Austin (2003) teorizaron acerca del aprendizaje por descubrimiento como metodología didáctica. Concretamente, fue Bruner (1961) quien más teorizó sobre el aprendizaje por descubrimiento en contraposición con el aprendizaje memorístico, es decir, promover la comprensión en lugar de la memorización de los conocimientos. Al igual que señala Camilloni (2007) la didáctica tiene que estimular a los estudiantes a comprender, para posteriormente, poder aplicar los conocimientos, por ello, la enseñanza por descubrimiento permite que, tras documentarse de un tema, se pueda descubrir una parte mayor de la realidad y, forjarse una opinión derivada de un conocimiento más profundo (Cálciz, 2011).

\section{OTROS ANTECEDENTES}

La elección de las temáticas de esta investigación no es casual. Se quería medir la implicación de los estudiantes en dos temáticas similares, pero diferenciadas. Por un lado, son similares porque tanto la situación de las personas refugiadas como la integración de los jóvenes latinoamericanos están englobadas en el ámbito de las migraciones y localizada en el contexto español. Además, son temas de interés social, lo cual los convierte en áreas objetivo de intereses para el marketing (Domegan et al., 2016). Por otro lado, pese a las similitudes, ambas temáticas tienen un matiz bien diferenciado. La integración de los jóvenes latinoamericanos es un tema latente, pero no de emergencia social, por lo que no está incluido en la agenda pública. Sin embargo, la situación de las personas refugiadas supuso una irrupción de la temática en la agenda pública, y, por ende, mediática del país (Crawley y Skleparis, 2018). Esto es relevante porque la influencia de las temáticas en la agenda pública es fundamental para que la sociedad se interese por la situación y decida investigar sobre la misma (Nava y Rueda, 2014), y derive en políticas públicas e intervenciones sociales Domegan et al., 2016).

\section{La situación de las personas refugiadas}

El 14 de diciembre de 1950 la Asamblea General de la ONU encargó la redacción de una Convención para regular la situación de los refugiados y los apátridas. Aquel encargo dio lugar a la adopción de la Convención de Ginebra, aprobada el 28 de julio de 1951 que se completa con el Protocolo de Nueva York aprobado el 31 de enero de 1967. En la actualidad son 148 los Estados que han ratificado alguno de estos dos instrumentos, de los cuales, 142 Estados han ratificado ambos. Un repaso por las fechas de ratificación de estos textos, por cada uno de los diferentes Estados, permite comprobar que en los procesos de transición democrática la firma de estos Tratados constituye un hito, es el caso de los países de la Europa del Este o de España. En España, la ratificación de esta Convención y su Protocolo se produjo en 1978, es decir, en los inicios del período democrático y se consagra, ese mismo año, en el artículo 13.4 de la Constitución. Además, la Carta de los Derechos Fundamentales de la Unión Europea recoge, en el artículo 18, la garantía del derecho de asilo. Po ende, los fundamentos legales para el reconocimiento del derecho de asilo y del estatuto del refugiado están sólidamente consolidados en España, así como en los países de la Unión Europea (UE) e, incluso, en la propia UE como institución.

En 2013, los países de la UE registraron un total de 431.000 solicitantes de asilo, este número aumentó a 627.000 en 2014, y alcanzó casi 1.3 millones en 2015. Esta última cifra es casi el doble del número de solicitudes recibidas en 1992 (672.000 en la UE cuando solamente la integraban 15 países, en lugar de los 28 actuales), que fue hasta este momento el récord de solicitudes de asilo, y que provenían principalmente de la ex Yugoslavia debido al comienzo de la Guerra de los Balcanes. En 2015, los solicitantes de asilo eran principalmente de Siria (29\%), Afganistán (14\%) e Irak (10\%) (Eurostat, 2016). Según datos del Fondo de las Naciones Unidas para la Infancia Internacional (Unicef), al menos 4.5 millones de niños habían sido desplazados de sus hogares a fines de 2015 debido a la inestabilidad en cinco países (Afganistán, Somalia, Sudán del Sur, Sudán y Siria), (Unicef, 2015). Además, más de 22.000 personas permanecen en campos de refugiados en Grecia, de las cuales casi 2.000 son menores no acompañados (Unicef, 2016). La mayoría de los que solicitaron asilo en 2015 tenían entre 18 y 35 años (53\%) y el 29\% eran menores de edad, de los cuales solo el 23,1\% estaban acompañados por un adulto (Eurostat, 2016). 
Para reconocer la condición de refugiado, en primer lugar, el país donde se solicitó el asilo debe realizar trabajos de protección (Hathaway, 2005), pero también desarrollar medidas que faciliten la integración de estas personas en sus nuevas sociedades de acogida (Ager y Strang, 2008). La UE se enfrenta actualmente a situaciones humanitarias y de emergencia en varios asentamientos de refugiados. Uno de los principales problemas es la duración de la estancia en los lugares de refugio, donde la duración de la llamada estancia temporal de refugiados es más larga de lo que debería ser (Davies y Batchelor, 2017).

\section{La integración de los jóvenes latinoamericanos en Madrid}

El tema de la integración social, cultural y económica de la segunda generación es un tema pendiente dentro de la academia. En España pocos trabajos abordan los aspectos relacionados con la construcción de la identidad de los adolescentes y jóvenes nacidos en España de padres extranjeros (segunda generación) (Borrego, 2003), y los nacidos en el extranjero y traídos al país a edad temprana (generación 1.5) (Portes et al., 2009). Existe la necesidad de conocer más sobre este segmento poblacional para poder diseñar políticas públicas que favorezcan su plena integración social, cultural y económica. En el contexto social actual las personas procedentes de grupos étnicos distintos han de desarrollar, para su plena integración social, una nueva identidad en los procesos de incorporación a otras sociedades que no son las de su origen por nacimiento. Para Isajiw (1990) la identidad de los adolescentes y jóvenes que se nutre de contextos diversos tiene un gran potencial de riqueza y capacidad de adaptación a entornos multiculturales. Esto representa una ventaja respecto a los adolescentes y jóvenes que han crecido en una única cultura.

En España dentro del marco de la integración social de los inmigrantes se habla de interculturalidad, que consiste en la promoción de un intercambio entre todas las culturas que coexisten. De este modo, se trata de un modelo de integración social que impulsa el respeto mutuo, el diálogo y la convivencia pacífica en un espacio público multicultural (Gonzálvez-Torralbo, 2016). A su vez, no hay que obviar el debate sobre la juventud transnacional. Se entiende por transnacionalismo al proceso por el cual los inmigrantes construyen campos sociales que comunican el país de origen y con el país de destino (Portes et al., 2003). La transnacionalidad, es una perspectiva teórica que ha acabado consolidándose en el estudio de las migraciones. Portes et al. (2003) denominan este fenómeno "globalización desde abajo". También existe una amplia base teórica sobre lo que ha supuesto la tecnología digital para la vida de la población migrante, especialmente en relación con la comunicación interpersonal transnacional (Huertas Bailén y Martínez Suárez, 2013). En la literatura sobre este tema existen conceptos ya consolidados como el de "presencia conectada" o la importancia de la figura de la madre transnacional y la familia; y los circuitos transnacionales (Gonzálvez-Torralbo, 2016).

Los adolescentes y jóvenes inmigrantes han sido a menudo protagonistas de conexiones transnacionales. Según Nilan y Feixa (2006), la conexión entre "hibridación", "mundos plurales" y "globalización", hace que las identidades generacionales en ocasiones estén deslocalizadas, aunque mantengan vínculos con lo local. En sus investigaciones hacen referencia a la "generación pachtwork" (marcada por los procesos de hibridación cultural), jóvenes que construyen su subjetividad a partir de los materiales híbridos provistos por culturas, consumos, resistencias, transnacionalismos y digitalismos globales o globalizados. El grado de integración de los jóvenes está determinado por las formas en la que los procesos afectan a su integración social, cultural y económica. Según Aparicio-Gervás y Delgado (2011), la integración es la capacidad de confrontar e intercambiar en una posición de igualdad, valores, normas y modelos de comportamientos. Para Pajares (2005) "es un proceso de equiparación de derechos de forma legal y efectiva, de las personas inmigradas con el resto de la población" (p.130). Por lo que es un proceso gradual y dinámico, así como también, bidireccional, que busca que los nuevos residentes lleguen a ser participantes activos en la vida económica, cívica y cultural del país de acogida.

\section{METODOLOGÍA}

El aprendizaje por descubrimiento se planteó en dos fases con dos investigaciones similares. En la primera fase, los estudiantes realizaron un estudio sobre la crisis de los refugiados derivada del conflicto en Siria, y tras el estudio de esa realidad, elaboraron una campaña de marketing denominada "Ponte en su lugar". El objetivo de esa campaña identificar a un público concreto, el denominado target en la ciencia del marketing, con la finalidad de que empatizasen con las personas que sufrían las consecuencias del conflicto bélico de Siria. En la segunda fase, en el curso académico siguiente con otros estudiantes diferentes, pero en la misma asignatura, se les indicó que realizase una investigación sobre los jóvenes latinoamericanos residentes en Madrid. Para ello, hicieron una revisión bibliográfica y posteriormente, participaron en el proceso de elaboración del cuestionario que implementaron con jóvenes latinoamericanos, lo cual les supuso un acercamiento directo con la población objeto de estudio.

La metodología que se ha seguido en esta investigación es cuantitativa. La técnica de producción de datos ha sido un cuestionario con 12 preguntas cerradas, en las que 10 de ellas eran medidas con una escala Likert de cinco valores, y las otras 2 preguntas eran de elección simple. Anteriormente, a la implementación 
del cuestionario, este instrumento se validó por un grupo de expertos del equipo docente e investigador de las áreas objeto de la investigación. Este cuestionario fue autocompletado por los estudiantes una vez finalizaron la actividad. En total, en las dos actividades se completaron 60 cuestionarios que es el $\mathrm{N}$ con el que se ha realizado el análisis de los datos. Una vez producidos los datos han sido codificados, lo que Bardín (1986) señala como "la trasformación que se realiza según reglas precisas de los datos brutos que por descomposición, agregación y numeración permite desembocar en una representación del contenido" (p. 74), para hacerlos más operativos para el análisis.

La técnica de análisis de datos utilizada ha sido un análisis de estadística descriptiva. Se hicieron varios tipos de análisis: univariante expresado en distribución de frecuencias, bivariante mediante tablas de contingencia, y multivariante por medio de análisis de correspondencias. Para estos análisis se utilizado el software estadístico Statistical Package for the Social Sciences (SPSS) en su versión 25. Una vez analizados los datos, se empleó el programa informático Excel de Microsoft Office, para la representación gráfica de los resultados más significativos para esta investigación.

\section{RESULTADOS Y DISCUSIÓN}

Los estudiantes de marketing tenían distintas opiniones sobre la crisis de Siria y la situación de los jóvenes latinoamericanos. La mayoría de las opiniones eran superficiales, pues casi ninguno conocía las problemáticas en profundidad. En consecuencia, había opiniones favorables a la solidaridad y otras opiniones en contra, pero, la mayoría forjadas sin mucho conocimiento, más allá del que uno puede formarse por los titulares de los medios de comunicación.

La valoración de las actividades era importante para medir el grado de aceptación de los estudiantes sobre las dos actividades (refugiados y jóvenes latinoamericanos). De hecho, la valoración de ambas acciones es muy favorable. De hecho, más dos terceras partes $(77,5 \%)$ de los estudiantes valoran las actividades como muy positivas o positivas; destacando la actividad de aprendizaje sobre la situación de los jóvenes latinoamericanos, que ha sido para el $29,8 \%$ de los estudiantes como una experiencia muy positiva. A su vez, cabe destacar como ningún estudiante valora como negativa o muy negativa la actividad de las personas refugiadas.

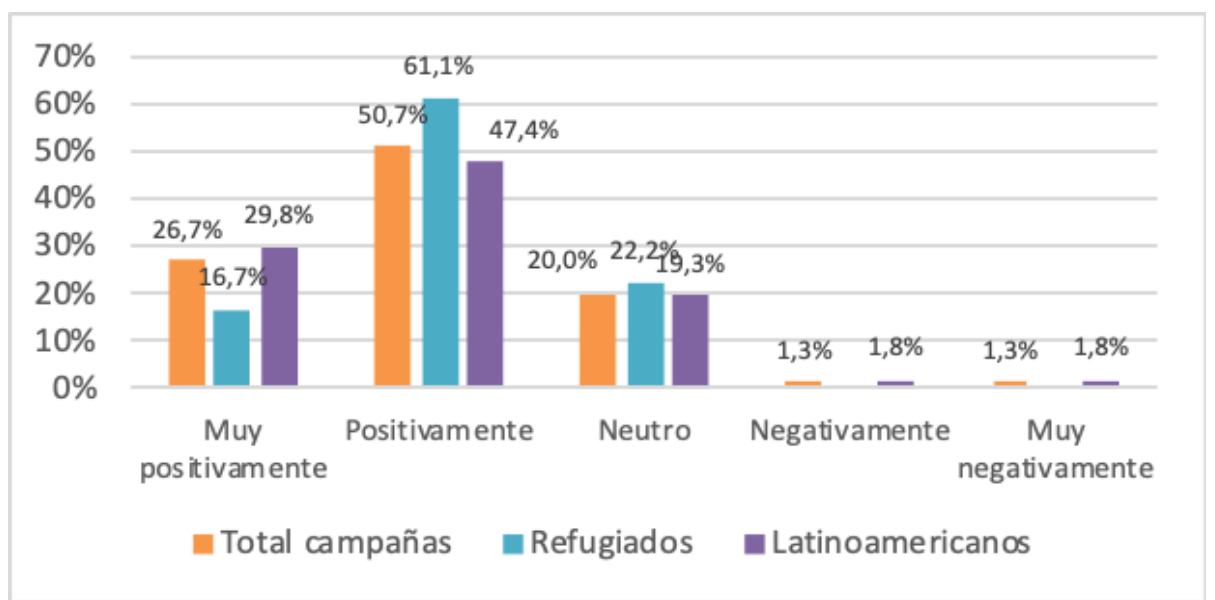

Fig. 1: Valoración del total y de las actividades de refugiados y jóvenes latinoamericanos.

Centrándonos en el punto que los estudiantes consideran de mayor aprendizaje, ellos señalan que los cuatro aspectos donde experimentaron mayor descubrimiento fueron: porque vieron que había muchas realidades diferentes $(50,0 \%)$; porque trataron con personas diferentes a su entorno habitual $(16,7 \%)$; por el trabajo que había detrás de la actividad (investigación previa de los estudiantes) (13,3\%); y porque se es más consciente de que se tiene (5,0\%). Únicamente, el 15\% de los estudiantes señalan que no experimentó descubrimiento por medio de las campañas, es destacable que en la campaña de jóvenes latinoamericanos este porcentaje aumenta hasta el $17,4 \%$, siendo de un $7,1 \%$ en la actividad de las personas refugiadas. Probablemente, este efecto se produzca porque la situación de los jóvenes latinoamericanos es más visible en la sociedad madrileña, que las personas refugiadas. En cuanto a la utilidad de las actividades, más de la mitad de los estudiantes (55,8\%) señalan que lo aprendido en las campañas es útil para su vida personal. Sin embargo, aquí encontramos diferencias en función de la actividad. En el caso, de las personas refugiadas la mitad de los estudiantes señalan que el aprendizaje se orienta hacia que hay que ayudar a los refugiados, y el $42,9 \%$ que es útil para su vida profesional; mientras que, en la campaña de los jóvenes latinoamericanos, casi dos tercios $(73,7 \%$ ) lo ven útil para su vida personal y un $15,8 \%$ le resulta útil para su 
vida personal. Esta diferenciación responde a que la temática sobre la situación de los refugiados despierta más los sentimientos de ayuda y colaboración humanitaria que el caso de los jóvenes latinoamericanos.

Respecto al marketing aplicado a campañas sociales -concretamente, de aspectos migratorios-, el $76,8 \%$ de los estudiantes creen que la utilidad del marketing radica en concienciar de las situaciones de vulnerabilidad. De hecho, creen que esta esta concienciación serviría para visibilizar las temáticas sociales (7,1\%). Analizando las campañas por fases, el $31,7 \%$ de los estudiantes señalan que la etapa de la campaña que valora más positivamente es la de concienciación social, seguida de la experiencia en el trabajo en equipo (28,6\%). De hecho, el trabajo en equipo aparece como rasgo positivo y enriquecedor en las variables de la investigación. Así como, se reitera lo apreciado en variables anteriores, es decir, que en la campaña de los jóvenes latinoamericanos se valora más la conciencia social $(39,2 \%)$ mientras que no se prima nada ( $0 \%$ ) en la campaña de refugiados; $y$, por el contrario, la ayuda a los refugiados se valora muy positivamente (16,7\%) y únicamente la ayuda de la campaña en la situación de los jóvenes latinoamericanos en el 3,9\%. A su vez, quienes valoran todas las fases de la actividad como positivas es mucho mayor en la acción de refugiados con casi un $60 \%$, mientras que es el $15,7 \%$ de los estudiantes de la actividad de los jóvenes latinoamericanos, quien valora todo como positivo.

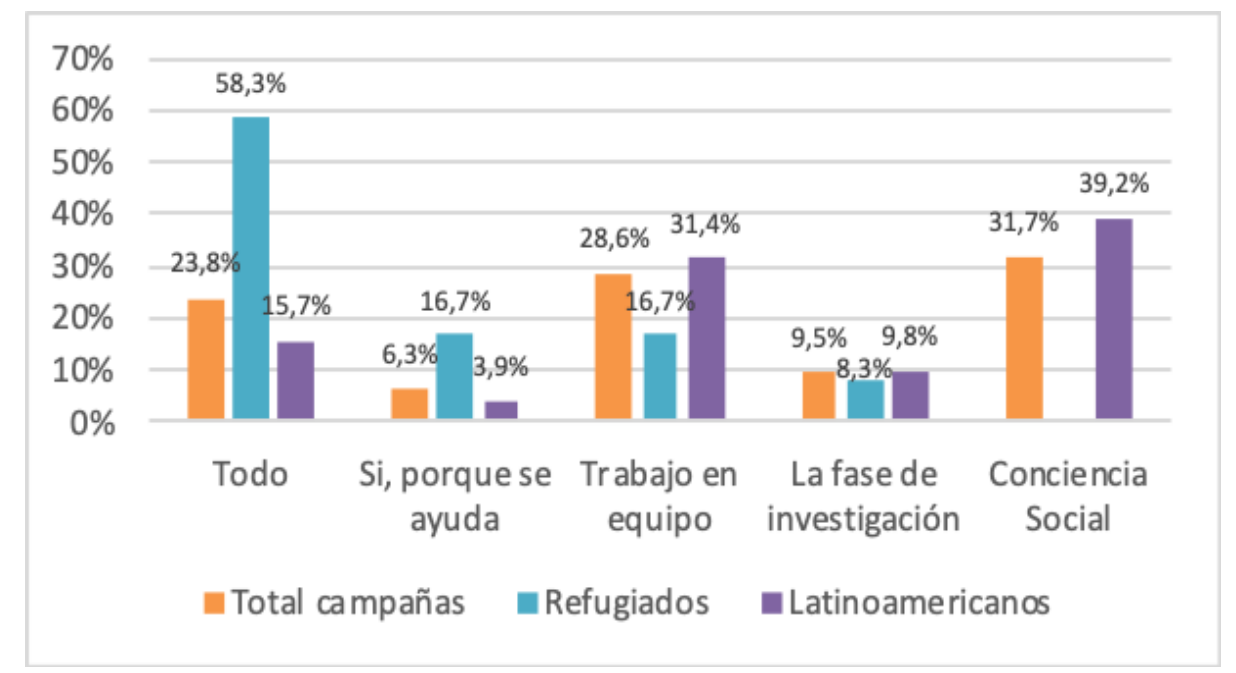

Fig. 2: Valoración de las fases del total y de las actividades de refugiados y jóvenes latinoamericanos.

Para finalizar el análisis univariante, las mejoras que realizarían los estudiantes de cara a futuras acciones sería aumentar las explicaciones sobre la temática a abordar $(30,2 \%)$, y más tiempo para desarrollar las acciones (14,3\%). Por otro lado, es reseñable como el $42,9 \%$ de los estudiantes no mejorarían nada de las campañas realizadas, lo cual es una valoración indirecta positiva de las actividades de aprendizaje por descubrimiento desarrolladas por los estudiantes.

En el análisis bivariante y multivariante, se observa que existe una relación entre la variable de valoración de las actividades y el descubrimiento de los estudiantes. En el análisis de correspondencias no solamente se mide la relación entre las variables, sino que se indica que categorías de las variables son las que se relacionan entre sí. Los estudiantes que valoran las actividades -la campaña de personas refugiadas y la campaña sobre los jóvenes latinoamericanos- como negativas o muy negativas están relacionados con quienes afirman que no han descubierto nada con estas actividades. Por el contrario, se muestra una relación entre quienes valoran muy positivamente la actividad y las personas que consideran que el aprendizaje por descubrimiento, lo han experimentado durante el trabajo en las campañas. La relación más fuerte a nivel estadístico se produce entre quienes valoran las campañas positivamente y, el descubrimiento, lo han tenido en crear conciencia sobre lo que ellos mismo tienen y en ver realidades diferentes de otras personas.

Tabla 1: Tabla de contingencia entre la valoración de las actividades y el aprendizaje por descubrimiento. * Chicuadrado $\mathrm{p}<0,036$

\begin{tabular}{|l|c|c|c|c|c|}
\hline & $\begin{array}{l}\text { El trabajo en cada } \\
\text { campaña }\end{array}$ & $\begin{array}{l}\text { Tratar personas } \\
\text { diferentes }\end{array}$ & $\begin{array}{l}\text { Ver realidades } \\
\text { diferentes }\end{array}$ & $\begin{array}{l}\text { Conciencia de } \\
\text { lo que tengo }\end{array}$ & No \\
\hline Muy positivamente & $37,5 \%$ & $30,0 \%$ & $33,3 \%$ & $33,3 \%$ & $0,0 \%$ \\
\hline Positivamente & $37,5 \%$ & $20,0 \%$ & $60,0 \%$ & $66,7 \%$ & $33,3 \%$ \\
\hline Neutro & $25,0 \%$ & $50,0 \%$ & $6,7 \%$ & $0,0 \%$ & $44,4 \%$ \\
\hline Negativamente & $0,0 \%$ & $0,0 \%$ & $0,0 \%$ & $0,0 \%$ & $11,1 \%$ \\
\hline Muy negativamente & $0,0 \%$ & $0,0 \%$ & $0,0 \%$ & $0,0 \%$ & $11,1 \%$ \\
\hline
\end{tabular}




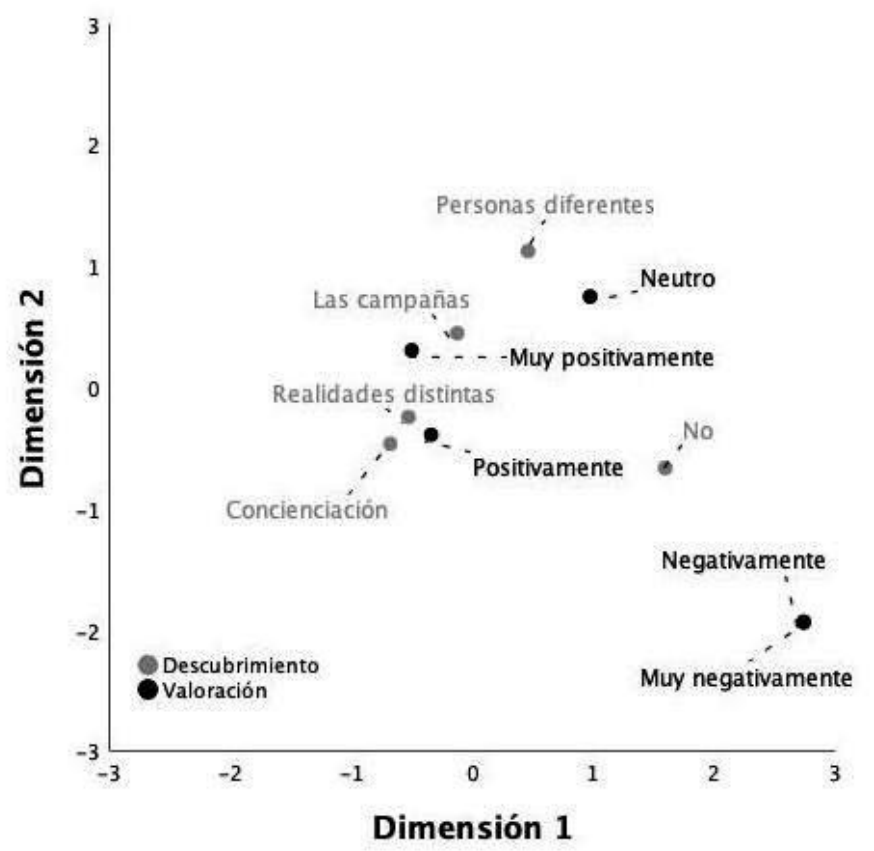

Fig. 3: Análisis de correspondencias entre descubrimiento y valoración.

El resultado de esta investigación es una experiencia colaborativa interdisciplinar que, como se ha visto en otros casos en distintas universidades, trata de superar los excesos de especialización y, enfoca la enseñanza universitaria de una forma integral gracias al intercambio de conocimientos para posibilitar la mejora de la enseñanza. La intervención de dos unidades académicas que representan áreas del conocimiento distintas como son, un departamento de investigación en ciencias sociales focalizado en la inmigración y los refugiados y, el claustro de profesores del Grado en Marketing de la Universidad Francisco de Vitoria cuya docencia es integral al combinar la formación en la ciencia del marketing junto a la enseñanza de las humanidades. Es relevante para este caso expuesto señalar que, esta universidad, tiene un planteamiento de enseñanza y educación que se define como "centrado en la persona", lo que, se traduce en que las asignaturas y líneas de investigación, se orientan siempre a dar un servicio integral a las personas. Esta visión de la enseñanza universitaria específica explica en parte que se haya desarrollado el caso expuesto en este artículo.

\section{CONCLUSIONES}

De acuerdo con los resultados de la investigación se pueden extraer las siguientes conclusiones válidas para los grupos de los dos cursos con los que se trabajaron las variables: aprendizaje por descubrimiento y valoración social de las campañas de personas refugiadas y jóvenes latinoamericanos: (1) Los estudiantes se manifestaron en las evaluaciones de esta enseñanza sorprendidos, al pasar en muchos casos de la indiferencia o prejuicio con este problema social, al aprendizaje de que los refugiados son personas como ellos y merecen su atención y ayuda; (2) Los estudiantes resaltaron su satisfacción de poder utilizar los conocimientos de marketing aprendidos en clase para ayudar en una causa social de máxima actualidad. Este caso demuestra que la realidad del mundo puede estar muy presente en un aula universitaria y, desde ésta, ofrecer propuestas valiosas a la sociedad. Con este hallazgo se corrobora lo que señalan Olate et al., (2011), Blom (2000) y Valle-Robles (2017), en el sentido de afirmar que el marketing al servicio de una causa social contribuye a la defensa de los derechos de las personas; Y (3) El aprendizaje por descubrimiento es una metodología de aprendizaje que conecta con los estudiantes y los motiva para investigar y estudiar causas sociales. Esta conclusión corrobora la teoría de Cálciz (2011) y Camilloni (2007) sobre que el aprendizaje por descubrimiento fomenta el interés y el posicionamiento de los estudiantes respecto a la temática investigada.

\section{REFERENCIAS}

Ager, A., y Strang, A. Understanding Integration: A Conceptual Framework. Journal of refugee studies, 21(2), 166-191 (2008).

Andreasen, A. R. Marketing Social Change: Changing Behavior to Promote Health, Social Development, and the Environment, 1-348, Jossey-Bass, San Francisco, CA (1995).

Aparicio-Gervás, J. M. y Delgado, M. Á. La educación intercultural en el Espacio Europeo de Educación Superior, 1-243 Itamut-Fified, (2011). 
Arias, W. Desarrollo de la historia de la psicología en el Perú. Revista Peruana de Psicología, 9 (1), 101-109 (2010).

Arias, W. Aprendizaje por descubrimiento vs. Aprendizaje significativo: Un experimento en el curso de historia de la psicología. Paulista de Psicología, 34 (87), 455-471 (2014).

Bardín, L. El análisis de contenido, 1-192, Akal, Madrid (1986).

Blom, J. Personalization a Taxonomy. CHI '00 extended abstracts on Human factors in computing systems, 313-314, The Hague, The Netherlands, ACM (2000).

Borrego, I. Los hijos de inmigrantes como tema sociológico: la cuestión de "la segunda generación". Anduli: revista andaluza deficiencias sociales, 3, 27-46 (2003).

Bruner, J. S. The act of discovery. Harvard Educational Review, 31, 21-32 (1961).

Cálciz, A. Metodologías activas y aprendizaje por descubrimiento. Revista digital innovación y experiencias educativas, 7, 1-11 (2011).

Camilloni, A. El saber didáctico. Buenos Aires: Editorial Paidós (2007).

Cova, B., y Badot, O. Marketing theory and practice in a postmodern era. In Marketing theory and practice (pp. 416-431). Palgrave, London, (1995).

Crawley, H., y Skleparis, D. Refugees, migrants, neither, both: categorical fetishism and the politics of bounding in Europe's 'migration crisis', Journal of Ethnic and Migration Studies, 44(1), 48-64, (2018).

Davies, S. y Batchelor, C. El reasentamiento como instrumento de protección para niños refugiados. Migraciones Forzadas, 54, 38-41 (2017).

Domegan, C., McHugh, P., Devaney, M., Duane, S., Hogan, M., Broome, B. J., y Piwowarczyk, J. Systems-thinking social marketing: conceptual extensions and empirical investigations. Journal of Marketing Management, 32 (11-12), 1123-1144 (2016).

Farieta, A. Conocimiento, descubrimiento y reminiscencia en el Menón de Platón. Universitas Philosophica, 30(60), 205234 (2013).

Gonzálvez-Torralbo, T. Las familias transnacionales ¿una tautología? Más allá de la dicotomía "distancia/proximidad geográfica”, Polis, 15 (43), 123-156 (2016).

Hathaway, J. The Rights of Refugees under International Law, 1-534, Cambridge University Press, Cambridge (2005).

Huertas-Bailén, A. y Martínez-Suárez, Y. Reflexiones en torno a la comunicación transnacional en el colectivo adolescente población migrante y apropiación de las TIC desde una perspectiva feminista. Revista TELOS, Cuadernos de Comunicación e Innovación, 96(37), 34-54 (2013).

Isajiw, W. Ethnic-identity retention; in R. Breton, W. Isajiw, W. Kalbach y G. Jeffrey Eds., 23-87, Ethnic-identity and equality. Toronto, University of Toronto Press (1990).

Kotler, P. Fundamentos de Marketing, 6ํㅡ. Ed. 1-96. Editorial Pearson Prentice Hall, España (2003).

Kotler, P. y Lee N. R. Social Marketing: Influencing Behaviors for Good. 3ํㅡ. Ed, 1-48, California, Sage Publications (2008).

Kotler, P. y Zaltman, G. Social marketing: an approach to planned social change, Journal of marketing, 35(3), 3-12. (1971). (1987).

Morales-Maure, L; García-Marimón, O; Torres-Rodríguez, A. y Lebrija-Trejos, A. Habilidades Cognitivas a través de la Estrategia de Aprendizaje Cooperativo y Perfeccionamiento Epistemológico en Matemática de Estudiantes de Primer Año de Universidad, Formación Universitaria, 11 (2), 45-65, (2018).

Nava, M., y Rueda, M. La evaluación docente en la agenda pública, Revista electrónica de investigación educativa, 16(1), 1-11, (2014).

Nilan, P. y Feixa, C. (eds) Global Youth? Hybrid Identities, Plural Worlds, 1-356, London \& New York, Routledge (2006)

Olarte, C.; Reinares, E. y Saco, M. Marketing de las causas sociales en Raya, Esther (coord.), Herramientas para el diseño de proyectos sociales, pp. 103-116, Material Didáctico, Trabajo Social: Buenos Aires (2011).

Pajares, M. La integración ciudadana, una perspectiva para la inmigración, 1-97, Barcelona, Ed. Icaria (2005).

Pérez, L. Marketing social: Teoría y Práctica. 1-568, México, Prentice Hall (2004).

Portes, A., Guarnizo, L. E. y Landolt, P. La Globalización desde abajo: Transnacionalismo inmigrante y desarrollo. La experiencia de Estados unidos y América Latina. FLACSO-México, FLACSO-Secretaría General (2003).

Portes, A., Aparicio, R., y Haller, W. La segunda generación en Barcelona: un estudio longitudinal. 1-233, Madrid, Centro de Estudios Migratorios de la Universidad Pontificia de Comillas (2009).

Sajid, S. Social Media and Its Role in Marketing. Business and Economics Journal, 7, 1-5 (2016).

Valle-Robles, J. E. Estrategias de mercadeo social en organizaciones sin ánimo de lucro del distrito de Santa Marta. Gestión, Competitividad e innovación, 353-364 (2017). 